\title{
Off-Diagonal Long-Range Order in Bose Liquids: Irrotational Flow and Quantization of Circulation
}

\author{
Gang $\mathrm{Su}^{1,2, *}$ and Masuo Suzuki ${ }^{2, \dagger}$ \\ ${ }^{1}$ Department of Physics, Graduate School at Beijing, University of Science and Technology of China, Chinese Academy of \\ Sciences, P.O. Box 3908, Beijing 100039, China \\ ${ }^{2}$ Department of Applied Physics, Faculty of Science, Science University of Tokyo, 1-3, Kagurazaki, Shinjuku-ku, Tokyo 162, \\ Japan
}

\begin{abstract}
On the basis of gauge invariance, it is proven in an elementary and straightforward manner, but without invoking any ad hoc assumption, that the existence of off-diagonal long-range order in one-particle reduced density matrix in Bose liquids implies both the irrotational flow in a simply connected region and the quantization of circulation in a multiply connected region, the two fundamental properties of a Bose superfluid. The origin for both is the phase coherence of condensate wave-functions. Some relevant issues are also addressed.
\end{abstract}

PACS numbers: 03.75.Fi, 67.40.-w, 05.30.-d

The discovery of superfluidity in liquid ${ }^{4} \mathrm{He}$ leads to London's proposal that the transition from He I phase to He II phase is an analog of Bose-Einstein condensation (BEC), namely, the superfluidity in He II is characterized by the macroscopic occupation of a single quantum state [1], despite that the liquid He II is actually a strongly interacting Bose system. On the basis of London's idea, Tisza [2], while Landau independently [3], developed phenomenologically a two-fluid model, in which the superfluid He II is regarded as being composed of superfluid and normal fluid components. The superfluid component is the single condensed quantum state, carrying no entropy, and thereby resulting in the assumption that the superfluid flow is irrotational. Meanwhile, Onsager (1) suggested that quantized vortex lines, or the quantization of vorticity according to Feynman independently [5], must exist in He II, which was experimentally verified a few years later [6]. Since then, the fact that the irrotational flow and the quantization of circulation (vortices) are two fundamental characters of superfluid He II is well established. Recently, the existence of vortices in a coherent collection of ultracold atoms has also been proposed and experimentally observed by spinning up the condensate [7]. On the other hand, Penrose and Onsager [8] generalized the mathematical description of BEC to interacting Bose systems, and manifested that BEC appears as the off-diagonal long-range order (ODLRO) in one-particle reduced density matrix exists, and liquid helium II was shown to possess such an order. Later, the concept was successfully extended to superconductivity in interacting fermion systems by Yang [9]. It is now well accepted that both superconductivity and superfluidity are quantum phases characterized by the existence of ODLRO. Nevertheless, what is the relationship between the fundamental properties of the condensates and the existence of ODLRO? The answer to the question for superconductors was presented a few years ago [10,11, i.e., the hypothesis of ODLRO in the two-particle reduced density matrix implies both Meissner effect and flux quantization, while the proof for superfluids is still absent.

In this paper, by noting the analog between superconductors and superfluids, we shall extend the proofs for superconductors presented in Refs. 10,11] to interacting Bose systems, and substantiate that the existence of ODLRO in one-particle reduced density matrix in a Bose liquid implies both irrotational flow and quantization of circulation. The origin for the two fundamental properties of a Bose condensate comes from the phase coherence of condensate wave-functions. Our proof does not invoke any ad hoc assumption.

Let us consider a bucket of Bose liquid composed of $N$ homogeneous interacting spinless particles, rotated with a constant angular velocity $\boldsymbol{\Omega}$. The Hamiltonian of the system is

$$
H=\sum_{j} \frac{1}{2 m}\left[\mathbf{p}_{j}+m \mathbf{v}_{s}\left(\mathbf{r}_{j}\right)\right]^{2}+\frac{1}{2} \sum_{j \neq l} V\left(r_{j l}\right),
$$

where $\mathbf{p}_{j}=-i \hbar \nabla_{j}$ is the momentum of $j$ th particle, $V\left(r_{j l}\right)$ denotes the interactions between particles, and $\mathbf{v}_{s}\left(\mathbf{r}_{j}\right)$ represents the drift velocity, given by

$$
\mathbf{v}_{s}(\mathbf{r})=\mathbf{\Omega} \times \mathbf{r}+\nabla \theta(\mathbf{r}),
$$

in which the first term comes from the Coriolis force, and the second term, to be identified below, originates from the gauge invariance. Eq. (2) comes out from the basic relation $\Omega=\frac{1}{2} \nabla \times \mathbf{v}_{s}$, as is in a classical case. The Schrödinger equation reads $H \psi_{n}\left(\mathbf{r}_{1}, \cdots, \mathbf{r}_{N}\right)=$ $E_{n} \psi_{n}\left(\mathbf{r}_{1}, \cdots, \mathbf{r}_{N}\right)$, where the orthonormal eigenfunctions $\psi_{n}$ 's are single-valued and symmetric. In analogy to the spirit of Refs. 10,11], we first make a gauge transformation. Under a small spatial displacement $\mathbf{r} \rightarrow \mathbf{r}-\mathbf{l}$, we have $\mathbf{v}_{s}(\mathbf{r}) \rightarrow \mathbf{v}_{s}(\mathbf{r}-\mathbf{l})=\mathbf{v}_{s}(\mathbf{r})+\nabla[\mathbf{l} \cdot(\boldsymbol{\Omega} \times \mathbf{r})+\theta(\mathbf{r}-$ l) $-\theta(\mathbf{r})]$, and $V\left(r_{j l}\right) \rightarrow V\left(r_{j l}\right)$. Then, the Schrödinger equation becomes

$$
H \tilde{\psi}_{n}\left(\mathbf{r}_{1}, \cdots, \mathbf{r}_{N}\right)=E_{n} \tilde{\psi}_{n}\left(\mathbf{r}_{1}, \cdots, \mathbf{r}_{N}\right)
$$

where 


$$
\tilde{\psi}_{n}\left(\mathbf{r}_{1}, \cdots, \mathbf{r}_{N}\right)=e^{i \frac{m}{\hbar} \sum_{j} \xi\left(\mathbf{r}_{j}, \mathbf{l}\right)} \psi_{n}\left(\mathbf{r}_{1}-\mathbf{l}, \cdots, \mathbf{r}_{N}-\mathbf{l}\right)
$$

is also the eigenfunctions of $H$, and the phase factor $\xi(\mathbf{r}, \mathbf{l})$ is defined by

$$
\xi(\mathbf{r}, \mathbf{l})=\mathbf{l} \cdot(\mathbf{\Omega} \times \mathbf{r})+\theta(\mathbf{r}-\mathbf{l})-\theta(\mathbf{r}) .
$$

Therefore, we have another set of orthonormal eigenfunctions, $\tilde{\psi}_{n}$ 's, for the system.

To gain insight into the underlying physics behind ODLRO in Bose liquids, we ought first to look at the intrinsic properties of the one-particle reduced density matrix $\rho_{1}\left(\mathbf{r}, \mathbf{r}^{\prime}\right)$. In accordance with the definition, $\rho_{1}\left(\mathbf{r}, \mathbf{r}^{\prime}\right)$ can be written down as

$$
\begin{aligned}
& \rho_{1}\left(\mathbf{r}, \mathbf{r}^{\prime}\right)=\int \cdots \int \frac{d \mathbf{r}_{2} \cdots d \mathbf{r}_{N}}{(N-1) !} \frac{1}{Z} \times \\
& \sum_{n} e^{-E_{n} / k_{B} T} \psi_{n}\left(\mathbf{r}, \mathbf{r}_{2}, \cdots, \mathbf{r}_{N}\right) \psi_{n}^{*}\left(\mathbf{r}^{\prime}, \mathbf{r}_{2}, \cdots, \mathbf{r}_{N}\right),
\end{aligned}
$$

where $Z=\sum_{n} e^{-E_{n} / k_{B} T}$, is the partition function. $\rho_{1}\left(\mathbf{r}, \mathbf{r}^{\prime}\right)$ is normalized as $\operatorname{Tr} \rho_{1}=\int d \mathbf{r} \rho_{1}(\mathbf{r}, \mathbf{r})=N$, and is single-valued. Now, we utilize the energy eigenfunctions given by Eq. (位) to represent $\rho_{1}\left(\mathbf{r}, \mathbf{r}^{\prime}\right)$ anew, and get the relation

$$
\rho_{1}\left(\mathbf{r}, \mathbf{r}^{\prime}\right)=e^{i \frac{m}{\hbar}\left[\xi(\mathbf{r}, \mathbf{l})-\xi\left(\mathbf{r}^{\prime}, \mathbf{l}\right)\right]} \rho_{1}\left(\mathbf{r}-\mathbf{l}, \mathbf{r}^{\prime}-\mathbf{l}\right) .
$$

Equation (7) illustrates that under a spatial displacement the one-particle reduced density matrix gains a position and displacement dependent phase factor. It is an intrinsic property of $\rho_{1}\left(\mathbf{r}, \mathbf{r}^{\prime}\right)$. On the other hand, we can express $\rho_{1}\left(\mathbf{r}, \mathbf{r}^{\prime}\right)$ in the spectral form as

$$
\rho_{1}\left(\mathbf{r}, \mathbf{r}^{\prime}\right)=\sum_{\nu} \lambda_{\nu} \Phi_{\nu}(\mathbf{r}) \Phi_{\nu}^{*}\left(\mathbf{r}^{\prime}\right)
$$

where $\Phi_{\nu}$ is the eigenfunctions of $\rho_{1}$, with eigenvalues $\lambda_{\nu}$. According to Penrose and Onsager [8], the existence of ODLRO in $\rho_{1}$ implies the spectral resolution such that

$$
\rho_{1}\left(\mathbf{r}, \mathbf{r}^{\prime}\right)=\lambda_{0} \Phi_{0}(\mathbf{r}) \Phi_{0}^{*}\left(\mathbf{r}^{\prime}\right)+\rho_{1}^{\prime}\left(\mathbf{r}, \mathbf{r}^{\prime}\right),
$$

where $\lambda_{0}$, on the order of $O(N)$, is the largest eigenvalue of $\rho_{1}, \Phi_{0}(\mathbf{r})$ is the corresponding eigenfunction (the socalled condensate wave-function), and $\rho_{1}^{\prime}\left(\mathbf{r}, \mathbf{r}^{\prime}\right)$ denotes other terms being on the order of $O(1)$. In the thermodynamic limit, $\rho_{1}^{\prime}\left(\mathbf{r}, \mathbf{r}^{\prime}\right) \rightarrow 0$ as $\left|\mathbf{r}-\mathbf{r}^{\prime}\right| \rightarrow \infty$. Comparing Eqs. (7) and (9) we may obtain under the limit $\left|\mathbf{r}-\mathbf{r}^{\prime}\right| \rightarrow \infty$ the following expression

$$
\Phi_{0}(\mathbf{r}) \Phi_{0}^{*}\left(\mathbf{r}^{\prime}\right)=e^{i \frac{m}{\hbar}\left[\xi(\mathbf{r}, \mathbf{l})-\xi\left(\mathbf{r}^{\prime}, \mathbf{l}\right)\right]} \Phi_{0}(\mathbf{r}-\mathbf{l}) \Phi_{0}^{*}\left(\mathbf{r}^{\prime}-\mathbf{l}\right) .
$$

With a careful analysis, one may find that this equation suggests

$$
\Phi_{0}(\mathbf{r})=e^{i \zeta(\mathbf{l})} e^{i \frac{m}{\hbar} \xi(\mathbf{r}, \mathbf{l})} \Phi_{0}(\mathbf{r}-\mathbf{l}),
$$

where a displacement dependent, but position independent phase factor, $\zeta(\mathbf{l})$ with $\zeta(\mathbf{0})=0$, may possibly emerge when extracting the information of individual function from the products of Eq. (10). As a result, we observe that the condensate wave-function, $\Phi_{0}(\mathbf{r})$, is mutually related by a phase factor at different positions. It is this property, being of fundamental importance, that enables us to prove the irrotational flow and the quantization of circulation in Bose superfluids.

In a simply connected region inside the superfluid, according to the rule governed by Eq. (11) we perform two opposite displacements, first $\mathbf{a}$ followed by $\mathbf{b}$, and then $\mathbf{b}$ followed by $\mathbf{a}$. Through comparing the relations satisfied by $\Phi_{0}(\mathbf{r})$ in the two operations, we discover that the phase factors satisfy the equation: $F(\mathbf{r}, \mathbf{a}, \mathbf{b})=F(\mathbf{r}, \mathbf{b}, \mathbf{a})$, with the function $F(\mathbf{r}, \mathbf{a}, \mathbf{b})=$ $\exp \left\{i \frac{m}{\hbar}[\xi(\mathbf{r}, \mathbf{a})+\xi(\mathbf{r}-\mathbf{a}, \mathbf{b})]\right\}$. By solving this equation, we have $\exp \left[i \frac{2 m}{\hbar} \boldsymbol{\Omega} \cdot(\mathbf{b} \times \mathbf{a})\right]=1$, yielding

$$
\boldsymbol{\Omega} \cdot(\mathbf{b} \times \mathbf{a})=n \frac{h}{2 m}, \quad(n=\text { integer }) .
$$

It is seen that the right-hand side of Eq. (12) is discretized, while the left-hand side can be continuously changed as the two operations can be arbitrarily choosen. The consistency condition requires

$$
\boldsymbol{\Omega}=\mathbf{0}
$$

This proves the property of the irrotational flow of the Bose condensate. However, we should stress that in terms of Eq. (2) the flow velocity, i.e., the superfluid velocity, should be in principle nonvanishing, i.e., $\mathbf{v}_{s}(\mathbf{r})=\nabla \theta(\mathbf{r})$, satisfying $\nabla \times \mathbf{v}_{s}(\mathbf{r})=0$.

In a multiply connected region inside the superfluid, there should be some singularities around which the fluid is rotating. These singularities are nothing but the vortex lines. In this case, we can repeat successively for infinitesimal displacements along a closed path $C$ which encloses at least one vortex line, and then pick up products of phase factors. Consequently, we have from Eq.(11) the relation $\Phi_{0}(\mathbf{r})=e^{i \frac{m}{\hbar} \oint_{C} \mathbf{v}_{s} \cdot d \mathbf{l}} \Phi_{0}(\mathbf{r})$, where use has been made of the single-valuedness of $\Phi_{0}(\mathbf{r})$, being a consequence of the single-valuedness of $\rho_{1}$, and $\exp \left[i \zeta\left(\oint_{C} d \mathbf{l}\right)\right]=\exp [i \zeta(\mathbf{0})]=1$. Therefore, we get

$$
\oint_{C} \mathbf{v}_{s} \cdot d \mathbf{l}=n \frac{h}{m}, \quad(n=\text { integer }) .
$$

This is the quantization of circulation, with the quantum of vorticity being $h / m$. If there is no any vortices encircled by the path loop $C$ in the multiply connected system, then the circulation will be identically zero, leading to again the property of irrotational flow everywhere away from the vortex cores.

The irrotational flow and the quantization of circulation have been well understood for superfluid He II based 
on the work of London, Onsager and Feynman quite long time before. Here we have offered another route, without ad hoc assumption, to understand the two fundamental characters of a Bose superfluid, and then established a deep connection between the basic properties of the condensate and the existence of ODLRO in Bose liquids. It is manifested that the condensate, as a single quantum state possessing these two essential features, is indeed characterized by the eigenstate of the one-particle reduced density matrix with the largest eigenvalue of the order of $O(N)$, as argued by Penrose and Onsager [8]. The preceding discussion shows that the phase coherence of condensate wave-functions is the origin for occurence of both properties.

Next, let us clarify the physical meaning of the quantity $\theta(\mathbf{r})$. In general, the condensate wave-function is assumed to be a complex number, and is usually denoted by

$$
\Phi_{0}(\mathbf{r})=\phi_{0}(\mathbf{r}) e^{i \alpha(\mathbf{r})},
$$

where the amplitude $\phi_{0}(\mathbf{r})$ and the phase factor $\alpha(\mathbf{r})$ are real functions. Define the condensate density as $\rho_{0}(\mathbf{r}) \equiv\left|\Phi_{0}(\mathbf{r})\right|^{2}=\left[\phi_{0}(\mathbf{r})\right]^{2}$. Then, the superfluid current density at position $\mathbf{r}$ is given by $J_{0}(\mathbf{r})=-\frac{i \hbar}{2 m}\left(\Phi_{0}^{*} \nabla \Phi_{0}-\right.$ $\left.\Phi_{0} \nabla \Phi_{0}^{*}\right)=\frac{\hbar}{m}\left[\phi_{0}(\mathbf{r})\right]^{2} \nabla \alpha(\mathbf{r})$. Since the current density and the superfluid velocity is related by $J_{0}(\mathbf{r})=$ $\rho_{0}(\mathbf{r}) \mathbf{v}_{s}(\mathbf{r})$, combining the irrotational property of the condensate we have

$$
\theta(\mathbf{r})=\frac{\hbar}{m} \alpha(\mathbf{r})
$$

As a result, we arrive at the conclusion that the superfluid velocity in the Bose liquids is proportional to the gradient of the phase factor of the condensate wave-function, namely

$$
\mathbf{v}_{s}(\mathbf{r})=\frac{\hbar}{m} \nabla \alpha(\mathbf{r}),
$$

a well-known result that was supposed for superfluid $\mathrm{He}$ II by London, Onsager and Feynman. However, our derivation shows that Eq. (17) holds true for any Bose system so long as the system possesses ODLRO in $\rho_{1}$. Thus, the phase of the condensate is closely related to the gauge invariance.

To explore the displacement dependent but position independent phase factor $\zeta(\mathbf{l})$, let us compare Eqs. (11) and (15). By considering (5) and (16) we can get $\tan [2 \alpha(\mathbf{r})]=\tan \left[2 \alpha(\mathbf{r}-\mathbf{l})+\zeta(\mathbf{l})+\frac{m}{\hbar} \mathbf{l} \cdot(\boldsymbol{\Omega} \times \mathbf{r})\right]$. Solving this equation and noting $\zeta(0)=0$, one may obtain

$$
\zeta(\mathbf{l})=2[\alpha(\mathbf{r})-\alpha(\mathbf{r}-\mathbf{l})]-\frac{m}{\hbar} \mathbf{l} \cdot(\boldsymbol{\Omega} \times \mathbf{r}) .
$$

This suggests that such a phase factor indeed exists if the condensate wave-function takes the form of (15). Consequently, we have from Eqs. (11) and (15)

$$
\phi_{0}(\mathbf{r})=\phi_{0}(\mathbf{r}-\mathbf{l}) .
$$

It shows that the amplitude of condensate wave-function, under assumption of the form (15), is displacementinvariant. This is convincing, because in superfluid $\mathrm{He}$ II, $\phi_{0}(\mathbf{r})$ is supposed as the square root of $n_{0}$, the macroscopic occupation number of the condensate, which is certainly translation-invariant. Here we have proved that for any condensed Bose system the condensate density possesses a translational invariance.

In the Bose condensate, the continuity equation becomes $\partial \phi_{0} / \partial t+\mathbf{v}_{s} \cdot \nabla \phi_{0}+(1 / 2) \phi_{0} \nabla \cdot \mathbf{v}_{s}=0$. After integrating it, we have

$$
\begin{aligned}
\frac{\partial}{\partial t} \int d \mathbf{r} \phi_{0}(\mathbf{r}, t)= & \frac{1}{2} \int d \mathbf{r} \phi_{0}(\mathbf{r}, t) \nabla \cdot \mathbf{v}_{s}(\mathbf{r}) \\
& -\int d \mathbf{r} \nabla \cdot\left[\mathbf{v}_{s}(\mathbf{r}) \phi_{0}(\mathbf{r}, t)\right]
\end{aligned}
$$

In view of Gaussian theorem, the second term of the right-hand side of above equation should be a surface integral, and is vanishing in the present case. For a steady flow, $\nabla \cdot \mathbf{v}_{s}(\mathbf{r})=0$, the above equation tells us that $\int d \mathbf{r} \phi_{0}(\mathbf{r}, t)$ is independent of time. If we define the spatial Fourier transform of $\phi_{0}(\mathbf{r}, t)$ as $\phi_{0}(\mathbf{k}, t)$, we know that $\lim _{\mathbf{k} \rightarrow 0} \phi_{0}(\mathbf{k}, t)$ is in fact independent of $t$. Consequently, the temporal Fourier transform of $\phi_{0}(\mathbf{k}, t)$, defined by $\phi_{0}(\mathbf{k}, \omega)$, has a property: $\lim _{\mathbf{k} \rightarrow 0} \phi_{0}(\mathbf{k}, \omega) \sim \delta(\omega)$. This implies that in the long wavelength limit there must be excitations whose frequency is zero in the Bose condensate. These massless excitations are nothing but Goldstone modes. As one knows, in He II the Goldstone bosons are identified as second sound. Therefore, by means of Goldstone's theorem one can conclude that in the Bose condensate the gauge symmetry is broken.

It is worth noticing that the irrotational flow and the quantization of circulation in a Bose superfluid can also be derived starting directly from Eqs. (15) and (17), as was done in previous literature. However, if we take a careful look, we can observe that the prerequisite of such reasonings is that the condensate wave-function was supposed to take the form of Eq. (15). Contrary to the previous arguments in which Eq. (15) was only an assumption without proof, the present derivation does not depend on what form of the condensate wave-function is taken, and only invokes the general principle of gauge invariance. In some sense, our result suggests implicitly that the condensate wave-function has the form of Eq. (15).

To this end, since superfluids and superconductors share some common features, it would be interesting to compare the correspondence between characters and variables of both, as summarized in the following table. 


\begin{tabular}{c|c}
\hline Superfluids & Superconductors \\
\hline Coriolis Force & Electromagnetic force \\
Angular velocity $(\boldsymbol{\Omega})$ & Magnetic field $(\mathbf{B})$ \\
Flow velocity $\left(\mathbf{v}_{s}(\mathbf{r})\right)$ & Vector potential $(\mathbf{A}(\mathbf{r}))$ \\
ODLRO in $\rho_{1}$ & ODLRO in $\rho_{2}$ \\
Irrotational flow & Meissner effect \\
$\left(\boldsymbol{\Omega}=\frac{1}{2} \nabla \times \mathbf{v}_{s}=0\right)$ & $(\mathbf{B}=\nabla \times \mathbf{A}=0)$ \\
Quantization of circulation & Flux quantization \\
$\left(\oint \mathbf{v}_{s} \cdot d \mathbf{l}=n \frac{h}{m}\right)$ & $\left(\oint \mathbf{A} \cdot d \mathbf{l}=n \frac{c h}{2 e}\right)$ \\
Broken gauge symmetry & Broken U $(1)$ gauge symmetry \\
Phase coherence & Phase coherence \\
BEC state of bosons & Condensate of Cooper Pairs \\
\hline
\end{tabular}

As is seen, the similarity between both systems is a consequence of ODLRO, or equivalently, of the broken gauge symmetry, which can be viewed as the profound underlying physics behind these two important systems in condensed matter, as emphasized by many authors (see, e.g. Ref. [12] for more references).

We close this paper with a few remarks in order.

(1) The present proof works for both noninteracting and interacting Bose systems. Thus, for an ideal Bose gas, as there is an ODLRO in $\rho_{1}$, the BEC state should also possess the property of irrotational flow and quantization of circulation. An ideal Bose gas is a superfluid, but it does not exhibit superfluidity, because it is unstable against any motion of the system. It is also manifested by Landau's criterion for superfluidity, i.e. the critical velocity is zero.

(2) For fragmented condensates, namely there are more than one eigenvalues of the one-particle reduced density matrix, $\rho_{1}$, of the order $O(N)$, leading to two or more eigenstates of $\rho_{1}$ being macroscopically occupied [13], our proof seems not to be sufficient, though the forms like Eq. (11) for condensates are still a possible solution. The fragmentation of condensates usually occurs in multi-component Bose systems, and cannot happen in single component systems, because it costs a macroscopic extensive exchange energy. For non-fragmented systems our argument is closely related to the so-called phase locking, as discussed by Nozieres [13].

(3) For the Bose systems with spin nonzero, the oneparticle reduced density matrix, $\rho_{1}\left(\mathbf{r}, \sigma ; \mathbf{r}^{\prime}, \sigma^{\prime}\right)$ with $\sigma$ and $\sigma^{\prime}$ spin indices, has a spectral resolution such that: $\rho_{1}\left(\mathbf{r}, \sigma ; \mathbf{r}^{\prime}, \sigma^{\prime}\right)=\lambda_{0} \Phi_{0}(\mathbf{r}, \sigma) \Phi_{0}^{*}\left(\mathbf{r}^{\prime}, \sigma^{\prime}\right)+\rho_{1}^{\prime}\left(\mathbf{r}, \sigma ; \mathbf{r}^{\prime}, \sigma^{\prime}\right)$, where $\rho_{1}^{\prime} \rightarrow 0$ for $\left|\mathbf{r}-\mathbf{r}^{\prime}\right| \rightarrow \infty$. Obviously, the condensate wave-function, $\Phi_{0}(\mathbf{r}, \sigma)$, is a spinor. In the absence of external magnetic fields, our reasonings remain similar to those for spinless systems. However, if a magnetic field is applied to the system, owing to a so-called "local spin-gauge symmetry" 14 there is a vorticity induced by spatial variations of the magnetic field, and the relation like Eq. (2) no longer holds, suggesting that our proof appears to be invalid, which is a possible consequence of the fact that the time-reversal symmetry is violated in the presence of an external field.
(4) For trapped alkali atoms like ${ }^{7} \mathrm{Li},{ }^{23} \mathrm{Na},{ }^{39} \mathrm{~K},{ }^{87} \mathrm{Rb}$ etc, the signature of BEC is successfully observed at extremely low temperatures (see, e.g. Ref. 15 for a review). These trapped Bose gases have finite sizes and are inhomogeneous, which contain the number of atoms typically from a few thousands to several millions. Therefore, the thermodynamic limit is not exactly reached for trapped gases, and consequently, the concepts of broken gauge symmetry and ODLRO appear, strictly speaking, not to be applicable in these finite-sized systems. However, though the phenomena occurred in trapped alkali atoms are not a thermodynamic phase transition in a rigorous sense, our preceding discussion might shed some useful light on it. For instance, the relation (11) satisfied by the condensate wave-function, $\Phi_{0}(\mathbf{r})$, which is normally considered as the order parameter, could also be used to investigate the linear, not rotational, motion of the condensate to a proper level of approximation. Quite recently, the irrotational flow and the quantization of vorticity in a BEC of ${ }^{87} \mathrm{Rb}$ atoms has been successfully observed at extremely low temperatures [16].

\section{ACKNOWLEDGMENTS}

One of authors (GS) would like to acknowledge Profs. H.T. Nieh, C.N. Yang and B.H. Zhao from whom he learned a great deal about concept of ODLRO. This work is also supported in part by JSPS, NSFC, State Key Project for Basic Research of China, and Chinese Academy of Sciences.

*E-mail: gsu@cc6.gsbustc.ac.cn.

${ }^{\dagger}$ E-mail: msuzuki@ap.kagu.sut.ac.jp.

[1] F. London, Nature 141, 643 (1938).

[2] L. Tisza, Nature 141, 913 (1938); Phys. Rev. 72, 838 (1947).

[3] L.D. Landau, J. Phys. USSR 5, 71 (1941).

[4] L. Onsager, Nuovo Cimento 6, Suppl. 2, 249 (1949).

[5] R.P. Feynman, in "Progress in Low Temperature Physics,", Vol. 1, ed. by C.J. Gorter (North-Holland, Amsterdam, 1955).

[6] H.E. Hall and W.F. Vinen, Proc. Roy. Soc. A 238, 204; 215 (1956).

[7] J.E. Williams and M. Holland, Nature 401, 568 (1999); M.R. Matthews et al, Phys. Rev. Lett. 83, 2498 (1999).

[8] O. Penrose, Philos. Mag. 42, 1373 (1951); O. Penrose and L. Onsager, Phys. Rev. 104, 576 (1956).

[9] C.N. Yang, Rev. Mod. Phys. 34, 694 (1962).

[10] G.L. Sewell, J. Stat. Phys. 61, 415 (1990); J. Math. Phys. 38, 2053 (1997).

[11] H.T. Nieh, G. Su and B.H. Zhao, Phys. Rev. B 51, 3760 (1995). 
[12] Bose-Einstein Condensation, eds. by A. Griffin, D.W. Snoke and S. Stringari (Cambridge University Press, Cambridge, 1995).

[13] P. Nozieres and D. Saint James, J. Phys. (Paris) 43, 1133 (1982). See also, P. Nozieres, in Ref. 12.

[14] T.L. Ho and V.B. Shenoy, Phys. Rev. Lett. 77, 2595 (1996); T.L. Ho, Phys. Rev. Lett. 81, 742 (1998).

[15] F. Dalfovo, S. Giorgini, L.P. Pitaevskii and S. Stringari, Rev. Mod. Phys. 71, 463(1999) and references therein.

[16] F. Chevy, K.W. Madison and J. Dalibard, Phys. Rev. Lett. 85, 2223 (2000). 